\title{
The European metrology network for climate and ocean observation
}

\author{
Emma Woolliams ${ }^{1}$, Céline Pascale $^{2}$, Paola Fisicaro ${ }^{3}$ and Nigel Fox ${ }^{1}$ \\ ${ }^{1}$ National Physical Laboratory (NPL), Hampton Road, Teddington, TW11 0LW, UK \\ ${ }^{2}$ Federal Institute of Metrology METAS, Lindenweg 50, 3003 Bern-Wabern, CH \\ ${ }^{3}$ Laboratoire national de métrologie et d'essais (LNE) 1 rue Gaston Boissier, 75015 Paris, FR
}

\begin{abstract}
EURAMET, the association responsible for coordinating the integrated metrology community in Europe, has recently established European Metrology Networks (EMNs) to support its aim to bring highquality scientific research and coordinated metrological services to meet the rapidly advancing needs of different stakeholders for metrology. The EMN for Climate and Ocean Observation is one of the initial six EMNs and has been set up as a European coordination body to support the global effort to bring rigorous metrological discipline into the observations that underpin our understanding of the climate and the oceans. This paper describes the network, its activity so far, and its plans.
\end{abstract}

\section{Introduction}

In 2015, responding to humanity's responsibility to limit and reverse our environmental damage while improving human health and quality of life, the United Nations ratified the "Sustainable Development Goals" (SDGs) [1]. The SDGs define humanity's joint hope for a future lived within ecological boundaries that has greater social equality, improved human health and improved quality of life. The SDGs are defined by 17 goals. Amongst these, goal 13 states: "Take urgent action to combat climate change and its impacts" and SDG 14 states: "Conserve and use the oceans, seas and marine resources for sustainable development".

Our present time is also the start of an information revolution as significant as the industrial revolution. The rapid increase in computational resources and mathematical tools for handling "big data", combined with the ever-increasing deployment of operational observational systems and the operational provision of environmental information services, such as the European Copernicus Services, have made information on the current (live), historical and predicted states of the environment available to everyone, at any time and in any place.

However, with an ever-increasing quantity of environmental information and observations, it is essential that these are underpinned by robust quality assurance. To identify a small climate trend from an observational record that is also sensitive to changes in weather, to seasonal effects, to geophysical processes, it is essential that observations are stable over multiple decades, while still allowing for changes in the observation instrumentation and operational procedures. The resultant data should also be traceable to a common reference, with well-understood uncertainty analysis, so that observations are interoperable and 
coherent; in other words, measurements by different organisations, different instruments and different techniques should be able to be meaningfully combined and compared.

It is here that metrology, the science of measurement, can contribute. Metrology has ensured that the SI units have been stable for nearly 150 years, are consistent worldwide and are coherent. Metrology has provided this consistency through the key principles:

1. Metrological traceability ([2],§2.41): Linking measurement results to a commonlyagreed primary reference (usually the international system of units, SI);

2. Comparison between independent measurement approaches: both exploratory scientific comparisons and formal comparisons that support the Mutual Recognition Arrangement [3], and

3. Uncertainty analysis following the principles of the Guide to the Expression of Uncertainty in Measurement [4].

These key principles of metrology can and should also be applied to the observations of ECVs and EOVs, albeit with necessary interpretation and adjustment, to provide quality assurance to the observations that society relies on.

In this paper we discuss the creation of a European Metrology Network for Climate and Ocean Observation. We recognise the considerable existing expertise in metrological methods in the scientific, commercial and operational communities that make and use observations, and the ongoing and growing existing collaboration between those communities and metrological institutes. However, in formalising and coordinating metrological activity (and resources) of the National Metrology Institutes (NMIs) in support of climate and ocean observation, we anticipate that this network will improve the engagement metrologists and domain-focussed experts. We see this network as the European contribution to a global effort to enhance the role of metrology in observations.

\section{Observing the climate and the ocean}

\subsection{Climate Observations}

The 2015 Paris Agreement seeks to limit the rise in temperature of the Earth to less than $2{ }^{\circ} \mathrm{C}$, with a target of $<1.5^{\circ} \mathrm{C}$, above pre-industrial levels to reduce the risks and impacts of climate change. Governments and international organisations need to make complex, inter-related decisions to meet this ambitious target. Such decisions require reliable climate models and a robust, integrated, global climate observing system.

The Global Climate Observing System (GCOS) is a programme, co-sponsored by the World Meteorological Organization, the Intergovernmental Oceanographic Commission of UNESCO, the United Nations Environment Programme and the International Council of Science, which defines itself [5] as working "towards a world where climate observations are accurate and sustained, and access to climate data is free and open". GCOS maintains definitions of, and requirements for, Essential Climate Variables (ECVs) [6]. ECVs are the core observations that should be made systematically, globally and robustly to observe the Earth's changing climate. These ECVs are used directly for a wide range of climate and commercial applications and are also used to validate and constrain climate models. At present there are 54 defined Essential Climate Variables, associated with three domains: atmospheric, oceanic and terrestrial. Each ECV has a set of observational requirements that cover the spatial and temporal resolution of the observation and the uncertainty required both for individual measurements and for trend observations (the latter called the "stability"). Many of these requirements are difficult to achieve in a laboratory, let alone in situ over 
decades. Furthermore, in most cases the ECVs are bio/geophysical in nature and obtained not through a direct observation, but through physical observables combined in a processing algorithm that often involves bio/geophysical models.

The GCOS website provides information about and locations of the observational records of ECVs that are openly accessible. These may come from in situ observations or from satellite remote sensing and include individual measurements and products that create (gap-filled) global information by merging observations with Earth system models, e.g. through meteorological reanalysis (data assimilation) or global/regional synthesis products. In almost all cases, ECV data records are produced by data processing workflows that transfer raw data into ECVs through multistep data processing. And further processing is required to create "climate data records" - long term records of ECVs that must be adjusted to account for instrument instability and instrument changes, as well as sampling issues.

14 of the 17 atmospheric ECVs, 6 of the 18 oceanic ECVs and 11 of the 16 terrestrial ECVs have a significant contribution from satellite Earth observation (EO). The others are observed primarily by in situ observational networks.

\subsection{Ocean Observations}

The oceans cover $71 \%$ of the Earth's surface. Oceans regulate the Earth's climate and have absorbed almost $93 \%$ of the enhanced anthropogenic greenhouse warming so far [7]; in doing this they have also been disproportionately impacted by climate change: sea levels have risen, changing coastlines, the oceans have become warmer, more acidic and with increased ocean stratification. These changes have altered ecosystems and increased the vulnerability of many marine species. Oceans are also a crucial source of food, water, energy and minerals for human life, and are a medium for transport ( $90 \%$ of goods are shipped by sea), recreation and commerce. The value of marine activities globally is about $5 \%$ of the global GDP, expecting to reach around US\$3 trillion by 2030 through sustainable growth (the value following an unsustainable scenario is smaller) [8]. The European Union's Integrated Maritime Policy focusses on 'Blue Growth' - harnessing the potential of Europe's oceans, seas and coasts to stimulate economic development within the environmental boundaries of the ocean ecosystems that sustain that growth.

To balance environmental, social and commercial concerns, the Global Ocean Observing System (GOOS), a programme executed by the Intergovernmental Oceanographic Commission (IOC) of UNESCO, has established a framework for ocean observing, centred around "Essential Ocean Variables" (EOVs). The EOVs include all the Essential Climate Variables in the oceanic domain and additional variables that relate to ecosystem, disasterwarning and commercial observational requirements.

\subsection{European observation programmes}

Europe has prioritised the provision of environmental information services through Copernicus - the European Union's Earth Observation Programme - which provides information from satellite and in situ observing systems to its users. Copernicus is coordinated and managed by the European Commission and implemented in partnership with the Member States, the European Space Agency (ESA), the European Organisation for the Exploitation of meteorological Satellites (EUMETSAT), the European Centre for Mediumrange Weather Forecasts (ECMWF), EU Agencies and Mercator Océan.

Copernicus includes data from its own set of dedicated operational satellites (the Sentinels) along with data from other commercial and public satellites and in situ networks. The Copernicus services transform this raw data into information by processing and 
analysing the data and making it available operationally, freely and openly accessible. Data are provided through six thematic streams: atmosphere; marine; land; climate change; security; and, emergency. The Copernicus Climate Change Service (C3S) provides observations of many of the ECVs in all three GCOS domains, while the Copernicus Marine Environment Monitoring Service (CMEMS) provides several of the EOVs. The Copernicus Atmosphere Monitoring Service (CAMS) covers both air quality measurements and climaterelevant ECV records and services and the Copernicus Global Land Services (CGLS) provides terrestrial products and services.

Through Copernicus and other observational monitoring programmes, Europe provides a significant contribution to the global climate observing system and the global ocean observing system. In the ocean domain, the new (established 2016) European Ocean Observing System (EOOS) is working to create a pan-European integrated ocean observing capacity. In the atmosphere, land and satellite domains, the European contribution is through global programmes such as the World Meteorological Organization's (WMO's) Global Atmosphere Watch (GAW) or the Group on Earth Observations (GEO) Global Earth Observation System of Systems (GEOSS) and the Committee on Earth Observation Satellites (CEOS).

\section{Metrological requirements for ECV and EOV observations}

The accuracy requirements specified by GCOS and GOOS for the ECVs and EOVs respectively are, in many cases, difficult to achieve in a laboratory but need to be made in the environment. To provide robust, interoperable and long-term data records of ECVs and EOVs, GCOS and GOOS must be underpinned by all the core metrological principles. At present, robust metrological methods are not routinely applied to many observations of ECVs and EOVs (with a few notable exceptions), but there is a growing recognition by the observation communities in the value that metrology can provide.

In 2010 the WMO and BIPM hosted a joint workshop on the "Measurement challenges for global observation systems for climate change monitoring". This recognised that many of the challenges faced by climate science are measurement challenges and that assessing climate change will depend crucially on the uncertainties associated with measurements and the robustness of the climate data [9]. The meeting recognised the existing activities of the observation community and welcomed the growing collaboration between the meteorology (and broader environmental) and metrology communities. It made several formal recommendations, including both general recommendations and targeted recommendations for specific needs. These included collaborations between communities to develop improved measurement standards and to perform comparisons, the establishment of metrological guidelines and operating procedures for observational networks, joint fundamental and applied scientific research and knowledge transfer to "better communicate the tools of metrologists" and "explain differences in the vocabularies used. Many of these recommendations have been implemented, but further work is needed.

The GCOS 2016 Implementation Plan [10] which set out the framework for improving the observation of the ECVs emphasises in its core list of actions the value of "improving collaboration ... [with] ... the metrological community" (p.37,38) and that a global climate observing system must have "documented traceability to SI standards" (p.65).

In this section we give some specific examples of the interaction between metrology and climate and ocean observation. This does not attempt to be an exhaustive list. 


\subsection{Atmospheric composition}

The WMO's Global Atmosphere Watch (GAW) Programme was established more than 25 years ago to improve our understanding of how humans are affecting atmospheric composition and to study the impacts of those changes. GAW provides systematic, global observations of the chemical composition of the atmosphere, along with work on the modelling required to connect these point measurements to global atmospheric models. GAW is based on the observations of networks of monitoring stations, along with supplementary observations from satellites and aircraft. The data are fed to world data centres and output as GAW products - greenhouse gas and ozone bulletins, as well as global data.

The GAW Implementation Plan for 2016-2023 [11] describes the quality assurance system in place, based around the Data Quality Objectives (DQOs) and the use of Central Calibration Laboratories (CCLs) and Regional Calibration Centres to provide traceability and comparability to the observational networks. The implementation plan describes a need to create consistency in methods and procedures and to ensure there are homogenized guidelines that include documentation of all uncertainties and use metrological vocabulary and uncertainty analysis techniques. The implementation plan also lists the core GAW partners and collaborating organisations, including the BIPM.

At present, national metrology institutes or designated institutes act as CCLs for four of the twenty variables being monitored by the GAW. Five variables have no CCL at present. The DQOs are often not met because of lack of suitable reference materials, lack of suitable reference data over the relevant pressure and temperature ranges or lack of instrumental transfer standards for chemically-reactive or otherwise difficult to prepare target components such as water vapour, NOx, NOy, black carbon and isotopic ratio signatures.

\subsection{Meteorological observations}

Well over a century ago, the world's meteorological institutes started making continuous and systematic observations of key meteorological parameters (including air temperature, humidity, pressure, wind speed and direction and precipitation). While these records were originally taken for weather forecasting applications, their true value is now realised in the form of a very long record of global temperatures and these, and similar, quantities are now defined as GCOS ECVs. WMO has a core purpose defined in its Convention to "promote the standardization of meteorological and related observations ..." and modern measurements are standardised through participation as part of the WMO Integrated Global Observing System (WIGOS).

As measurement technologies and methodologies evolve, there can be systematic step changes in records from individual meteorological stations. Such changes occurred, for example in the early 20th Century with the introduction of the Stevenson Screen and in the late 20th Century with a transition to automated measurements. When aggregated, these changes result in spurious changes in global and regional meteorological records which must be corrected to accurately reconstruct a faithful climate record. Anticipating future changes, GCOS and the WMO Commission for Climatology requested a group of scientists to develop an outline for a global land surface climate fiducial reference measurement network that would consist of reference stations that meet ideal conditions for instrumentation, calibration procedures and location. This group's report [12] emphasised the importance of establishing metrological traceability, comparability and representativity through implementing metrological best practices. A similar approach has been successfully implemented in the United States of America by the US Climate Reference Network. After 19 years of operation 
the USCRN has substantially validated the procedures used in analyse the historical meteorological data, and thus added confidence to these historical estimates.

In terms of upper air meteorological measurements, a GCOS report in 2007 [13] identified shortcomings in the design and implementation of the existing upper-air measurement network which greatly limited the accuracy and detail of observations needed to specify how climate has varied and changed above the Earth's surface. The report established the requirements for the GCOS Reference Upper-Air Network (GRUAN) to provide reference quality measurements of upper air ECVs. The GRUAN network was established in 2008 with the primary goals of providing vertical profiles of reference measurements suitable for reliably detecting changes in global and regional climate on decadal time scales, initially for temperature, pressure and water vapour, with future extension to other atmospheric ECVs. GRUAN measurements aim to provide:

- a traceable reference standard for global satellite-based measurements of atmospheric ECVs,

- ensure that potential gaps in satellite measurement programmes do not invalidate the long-term climate record,

- a reference standard for the measurements made within the existing GCOS UpperAir Network (GUAN),

- full characterization of the properties of the atmospheric column.

- metrologically-traceable uncertainties for all GRUAN-certified observations.

These goals have been agreed to by GCOS and WMO. As of May 2019, GRUAN comprises of 26 sites, 12 of which have been GRUAN certified [14], with the eventual goal of GRUAN being a global network of 30-40 measurement sites, which to the extent possible, build on existing observational networks and capabilities.

\subsection{Ocean observation}

The marine communities already collaborate in national, European and international organisations, usually covering several EOVs. Generally, the development of measurement systems and observational infrastructures are well coordinated, essentially because these structures are multidisciplinary and interconnected and must deliver data serviceable to every organisation member and to policy-makers. However, this integrated landscape is lacking a multidisciplinary and coordinated approach to providing metrological traceability, comparison and uncertainty analysis to the EOV observations. In recent years, ocean observation infrastructures have grown considerably and today they are capable to deliver thousands of measurements per second the metrological quality of which is crucial to their usability. Therefore, the oceanographic community is increasingly seeking the co-operation of metrology institutes.

In this frame, the European community is establishing a community-driven coordinating framework: the European Ocean Observing System (EOOS), in response to the observation needs for both environmental and economic activities and decisions. The EOOS Strategy and Implementation Plans $[15,16]$ describe the importance of involving metrologists in developing "best practice" for the "systematic harmonisation of ocean observing in Europe". The European Union's Joint Programming Initiative: "Healthy and Productive Seas and Oceans" (JPI-Oceans), wrote in its report on the need for EOOS [17], that “... to be useful for research and decision-making at a transnational level, all the incoming data have to be comparable and amenable to fitness-for-purpose assessments in relation to specific usergroup requirements. This will require measurements to be metrologically referenced ..." 
At present, such metrological referencing is limited for some EOVs. The JPI-Oceans report states: "although a few recognized standards are in place, no certified reference material is available ... to this end, the National Metrology Institutes can contribute by helping the oceanographers and manufacturers to establish validated metrological procedures".

To ensure data quality, oceanographic institutes are increasingly implementing metrological principles in their quality assurance systems. In some cases, they are considering embedding the quality assurance of EOV measurements into existing national accreditation systems that, in turn, are embedded in European and international accreditation structures like the European Accreditation (EA) and the International Laboratory Accreditation Cooperation (ILAC). Institutions seeking accreditation for the measurement of specific EOVs would have to give evidence that their measurements are performed in compliance with international (written) standards, e.g. ISO/IEC 17025 or ISO 22013, which include metrological requirements like traceability and adequate uncertainty calculations explicitly. Besides some typical physical measurement quantities also measured in other areas, accreditation bodies are rarely prepared to cover EOVs that are based on measurement procedures specific to oceanography. As a consequence, oceanographic institutes, accreditation bodies and respective standardisation bodies seek metrological support on those issues.

\subsection{Satellite Earth Observation and land observations}

The Committee on Earth Observation Satellites (CEOS) is the effective space arm of the intergovernmental Group on Earth Observations (GEO). A central part of GEO's mission is to build the "Global Earth Observation System of Systems" (GEOSS). It is crucial for the implementation of GEOSS that data "are accessible, of identified quality and provenance and interoperable" [18]. In establishing the principles to achieve this, CEOS held several workshops and in 2008 formally endorsed the Quality Assurance Framework for Earth Observation (QA4EO) [19] as a documented means to achieve the desired interoperability. QA4EO is established around a guiding principle that "all data and derived products must have associated with them a quality indicator based on documented quantitative assessment of its traceability to a community-agreed reference standard". The QA4EO guidelines that support this were written in collaboration between the Earth Observation and metrology communities, with NPL and NIST scientists developing the guidelines and helping translate these into concepts more suitable for Earth observation communities. For example, the QA4EO guideline on comparisons (guideline 4) was based on the metrology community's mutual recognition arrangement processes and, in particular, on the CCPR guidelines for comparisons [20].

The uptake of the concepts of QA4EO in the CEOS community has grown rapidly since 2008 as it has gained increasing recognition. The European Space Agency now explicitly requires the implementation of QA4EO principles in many of its processes. There have also been many European research projects (funded by the space agencies ESA and EUMETSAT and by the European Union's Horizon 2020 programme and its predecessor) that have involved collaboration between metrologists and Earth observation experts to develop methods for implementing QA4EO into each community. This has led to guidelines for developing and reviewing climate data records of ECVs [21], to the establishment of "fiducial reference measurements" (FRM) of SI-traceable ground truthing observations (e.g. [22]), and subsequent "FRM-networks" and to the development of guidelines for applying metrological techniques to fundamental climate data records (the basic satellite product that is used to 
generate different ECVs) [23]. Recently, there has been approval and anticipated funding to design, build and launch a satellite that puts a national metrology institute into orbit, implemented through the ESA Earth Watch Programme. The TRUTHS satellite [24] will provide both a climate benchmark by making the most accurate observations of incoming and reflected solar radiation, and will serve as an inflight calibrator, able to transfer its SItraceability to other sensors in orbit.

\section{The creation of the European Metrology Network for Climate and Ocean Observation}

The European Association of National Metrology Institutes (EURAMET) has recently established European Metrology Networks (EMNs) to support its vision to ensure that Europe's metrology capability, based on high-quality scientific research and a long tradition of valuable international cooperation, meets the rapidly-advancing needs of end users. EMNs will analyse the European and global metrology needs and address these both in coordinating the research and services of European metrology institutes and in providing a single point of contact for information, to underpin regulation and standardisation and to promote metrological practice. The European Metrology Network for Climate and Ocean Observation is one of the first six EMNs to be established by EURAMET. Further EMNs will be established over the next few years, with an expectation of there being around 15-20 EMNs eventually.

The EMN for Climate and Ocean Observation signed its Memorandum of Understanding in May 2019 and held its first meeting in June. The EMN is organised with three technical sections. Each section covers the metrological contribution to support those making and using climate and oceanographic observations within the thematic area of the section. The thematic areas broadly correspond to the divisions of the GCOS ECVs and principal observational techniques. The authors of this paper are the chair of the network and the co-chairs heading each section respectively (elected for the period 2019-2022).

The Atmosphere Observation Section covers the metrological contribution to support in situ surface, upper air and composition measurements of the GCOS Atmospheric ECVs. These include measurements of gases, aerosols, water vapour and properties of clouds, along with physical ECVs such as temperature, pressure and wind speed and direction.

The Ocean Observation Section covers the metrological contribution to support in situ measurements of GCOS Oceanic ECVs along with the broader GOOS EOVs. These include physical (e.g. temperature, salinity, currents, ice), biogeochemical (e.g. dissolved gases and nutrients, acidification, particulate matter and tracers) and biological (e.g. phytoplankton, ocean sound) variables. The Ocean Observation Section reflects the broader variety of economic, social, and environmental perspectives relating to the oceans, alongside the climate applications.

The Land and Earth Observation Section covers the metrological contribution to support in situ measurements of the GCOS terrestrial ECVs (hydrology, cryosphere, biosphere and human resource use), along with the remote sensing (satellite, aircraft, drones and ground-based remote sensing) observations of ECVs in all three GCOS categories: Land, Ocean and Atmosphere. The scope of activities includes standards and methods for the calibration of land networks and pre-flight, in-flight and post-launch calibration of remotely deployed sensors as well as the metrology needed to assign quality metrics to derived information products, i.e. accounting for retrieval algorithms.

European national metrology institutes and designated institutes (i.e. members of EURAMET) can become members of the EMN, while any other interested organisation is welcome to become a partner of the EMN. 


\section{Expected outputs and outcomes of the EMN for Climate and Ocean Observation}

The EMN for climate and ocean observation has an initial workplan with the following outputs:

1. An initial membership consisting of 20 metrology institutes in Europe and $5+$ partner institutes.

2. A stakeholder needs review and report (written 2020, updated 2022) that describes the stakeholder needs for metrology in the observation and use of ECVs and EOVs, based on semi-structured interviews with stakeholders identified in a "snowball" stakeholder identification process and prioritised through stakeholder mapping, along with two stakeholder workshops in 2020 and 2022.

3. The establishment of a single point-of-contact for metrology support for climate and ocean observation with a website that presents European metrological capability in a user-centred manner (2020), and with the conceptual framework for a future "one-stop-shop" describing the long-term operation of the EMN (2023)

4. A strategic research agenda that identifies the core research requirements for European metrology and coordinates that research activity to best meet the needs of stakeholders (2021 and biannually thereafter)

5. A collated set of training material to teach the observation communities the principles of metrology (2019, updated thereafter).

6. A webpage and communications material to present the concepts of metrology to different stakeholder communities (2019, updated thereafter).

From these outputs, we expect to achieve the following outcomes:

- The establishment of metrological principles at the heart of in situ and spacebased observational networks, which meet the user requirements of interoperability, multi-decadal stability and global consistency.

- Improved SI-traceable measurement techniques and reference standards for in situ and satellite observations.

- The extension of the involvement of European metrologists in observational networks - moving from the provision of traceability to involvement in processing chains, validation and modelling.

- A long-term sustainable European infrastructure to ensure that metrology is aware of, predicts and responds to evolving stakeholder needs and which enables metrology to have significant impact in the field of climate and ocean observation.

- Greater coordination of European metrological research to maximise crossfertilisation of ideas and concepts, to encourage cross-disciplinary working amongst metrologists (and perhaps, through metrologists, of the observational networks themselves) and to use the European national and international metrological funding for the whole to be more than the sum of the parts.

- The development of the skills of a greater number of scientists providing them with expertise in both observations and metrology.

- An improved metrological basis to the Copernicus environmental information services which support both climate and broader economic and societal applications. The Copernicus economic benefit to end users is $\sim 3$ b€ 
(2008-2020) and growing [25], and the global ocean economy is $\sim 5 \%$ of global GDP.

\section{Conclusions}

EURAMET has established a European Metrology Network for Climate and Ocean Observation to provide a single point-of-contact for European metrology in supporting the creation and use of observations. It is hoped that this network will accelerate the already significant role that metrologists are playing in ensuring that observations are interoperable, have multi-decadal stability and are internationally consistent.

Understanding climate change and the sustainable use of the oceans are not problems that can be seen in regional isolation and, therefore it is important to recognise that this European effort is in support of a broader international effort. We recognise similar efforts of our sister metrology institutes in other regions of the world and hope that there will be further collaboration between the regions.

Acknowledgements: We thank the Euramet team, particularly Anne Trumpfheller and Thomas Damitz, as well as Maguelonne Chambon for help with setting up the network, and Ruchi Mathur for her management of the project as well as all the European metrology institutes who have already become active members of the network. Steffen Seitz, Tom Gardiner and Michael de Podesta have provided valuable feedback on drafts of this paper. This project (18NET04) has received funding from the EMPIR programme co-financed by the Participating States and from the European Union's Horizon 2020 research and innovation programme.

\section{References}

1. www.un.org/sustainabledevelopment/

2. VIM: International Vocabulary of Metrology - Basic and General Concepts and Associated Terms (VIM 3rd edition), JCGM 200:2012, Sevres, Paris; (2012); www.bipm.org

3. CIPM: Mutual Recognition of National Measurement Standards and of Calibration and Measurement Certificates Issued by National Metrology Institutes, Sevres, Paris (14 October 1999); www.bipm.org

4. GUM : JCGM 100:2008 Guide to the expression of Uncertainty in Measurement Sevres, Paris; (2012); www.bipm.org

5. https://gcos.wmo.int/en/home

6. S. Bojinski, M. Verstraete, T.C. Peterson, et al. Bulletin of the American Meteorological Society. 95, 1431 (2014)

7. IPCC Climate Change 2013: The Physical Science Basis. Contribution of Working Group I to the Fifth Assessment Report of the Intergovernmental Panel on Climate Change. Cambridge University Press, Cambridge, United Kingdom and New York, NY, USA, 1535 (2013) ; www.ipcc.ch/report/ar5/wg1/

8. OECD : The Ocean Economy in 2030, OECD Publishing, Paris. (2016); DOI : 10.1787/9789264251724-en 
9. BIPM, WMO, IOM : Report on the WMO-BIPM workshop on Measurement challenges for global observation systems for climate change monitoring. Rapport BIPM-2010/08 ; WMO/TD-No. 1557, IOM-Report No. 105 ; (2010)

10. GCOS, The Global Observing System for Climate: Implementation Needs; GCOS200, GOOS-214; World Meteorological Organization. (2016).

11. WMO. WMO Global Atmosphere Watch (GAW) Implementation Plan: 20162023. ISBN: 978-92-63-11156-2 (2016).

12. PW. Thorne, HJ. Diamond, B. Goodison et al. Int J Climatol. 38. 2760-2774 (2018)

13. GCOS. GCOS reference upper-air network (GRUAN) : Justification, requirements, siting and instrumentation options. GCOS-112 (2007)

14. www.gruan.org

15. EOOS. EOOS Strategy 2018 - 2022, www.eoos-ocean.eu, (2018)

16. EOOS. EOOS Implementation Plan 2018 - 2022, www.eoos-ocean.eu (2018)

17. JPI Oceans. Joint Action Factsheet on European Marine Sensor Calibration Network. www.jpi-oceans.eu/european-marine-sensors-calibration-network (2017)

18. www.earthobservations.org/geoss.php

19. GEO. "A quality assurance framework for Earth Observation, Principles", www.qa4eo.org; (2010)

20. CCPR. Report CCPR-G2: Guidelines for CCPR key comparison report preparation. www.bipm.org/en/committees/cc/ccpr/publications-cc.html (2019)

21. J. Nightingale, J.P.D. Mittaz, S. Douglas, et al. Remote Sensing, 11(8), 986. (2019)

22. www.frm4sts.org and frm4soc.org and www.frm4alt.eu

23. J. Mittaz, C.J. Merchant, E.R. Woolliams, Metrologia, 56, 032002. (2019)

24. N. Fox, A. Kaiser-Weiss, W. Schmutz et al, Phil. Trans. R. Soc A, 369, 4028-4063 (2011)

25. Copernicus. Copernicus Market Report, Nov 2016. 